\title{
A stability criterion for elasto-viscoplastic constitutive relationships
}

\author{
F. Pisanò ${ }^{1, * \dagger}$ and C. di Prisco ${ }^{2}$ \\ ${ }^{1}$ Department of Geoscience and Engineering, Delft University of Technology, Delft, Netherlands \\ ${ }^{2}$ Department of Civil and Environmental Engineering, Politecnico di Milano, Milan, Italy
}

\begin{abstract}
SUMMARY
In this paper, the onset of mechanical instability in time-sensitive elasto-viscoplastic solids is theoretically analyzed at the constitutive level and associated with the occurrence of 'spontaneous accelerations' under stationary external perturbations. For this purpose, a second-order form of Perzyna's constitutive equations is first derived by time differentiation, and a sufficient stability condition is identified for general mixed loading programs. These loading conditions are in fact the most general in both laboratory tests and real boundary value problems, where a combination of certain stress and strain components is known/prescribed.

The theoretical analysis leads to find precise stability limits in terms of material hardening modulus. In the case of constitutive relationships with isotropic strain-hardening, no instabilities are possible while the hardening modulus is larger than the so-called 'controllability modulus' defined for (inviscid) elasto-plastic materials. It is also shown that the current stress/strain rate may also directly influence the occurrence of elasto-viscoplastic instability, which is at variance with elasto-plastic inviscid media. Copyright (C) 2015 John Wiley \& Sons, Ltd.
\end{abstract}

Received 27 January 2015; Revised 23 April 2015; Accepted 28 April 2015

KEY WORDS: viscoplasticity; Perzyna; rate-dependence; stability; controllability; mixed loading

\section{INTRODUCTION}

Modeling and predicting failure phenomena in solid media is of utmost importance in many applied and engineering sciences. Meaning the concept of 'failure' in its broadest sense, the collapse of both natural and man-made systems can be induced by a wide variety of failure/instability processes at the material level. This statement especially applies to geomaterials [1-4]: indeed, these are characterized by complex granular structures with either loose or interconnected grains, they interact with interstitial fluids, and may suffer degradation and fracture processes caused by mechanical, hydraulic, thermal, and chemical solicitations. As a consequence, defining a priori the whole range of situations under which geomaterials 'fail' is not trivial and is still intensively discussed within the scientific community $[5,6]$.

In the context of continuum-based theories, most approaches for the inelastic analysis of solids and structures have been developed in the framework of rate-independent (or inviscid) plasticity [7-10], that is, under the assumptions that (i) unrecoverable deformations take place instantaneously and (ii) no role is played by the external perturbation rate. Although elasto-plasticity has been proven to capture most features of the inelastic response of geomaterials, the assumption of rateindependence prevents some important experimental evidences to be reproduced, such as creep and relaxation processes.

\footnotetext{
*Correspondence to: F. Pisanò, Department of Geoscience and Engineering, Delft University of Technology, Delft, Netherlands.

${ }^{\dagger}$ E-mail: f.pisano@tudelft.nl

Copyright (C) 2015 John Wiley \& Sons, Ltd.
} 
To overcome this intrinsic limitation of standard elasto-plasticity, the theory of elastoviscoplasticity was purposely introduced. Although different viscoplastic approaches are available in literature [11-15], viscoplastic models are all based on the concept of 'delayed plastic flow', implying that a finite amount of time is required for irreversible (viscoplastic) strains to develop. As a result, the time variable actively contributes to the global material response, which is in turn determined by the interaction between the intrinsic material rate-sensitiveness and the external loading rate. In what follows, the most widespread viscoplastic framework introduced by [13] will be exclusively considered.

The experimental investigation of rate effects in geomaterials has led to regard elastoviscoplasticity as a suitable framework for conceiving constitutive relationships [16-20] and reproducing certain material instabilities [21-23]. In addition to experimental motivations, viscoplasticity has also gained further popularity as a simple regularization technique in finite element computations, because it mitigates the mesh-dependence effects arising from bifurcated responses (e.g., strain localization) [15, 24-26].

In the light of the aforementioned premises, the stability analysis of viscoplastic constitutive equations is needed to assess the following: (i) the suitability of viscoplastic models for geomaterials and (ii) the reliability/objectivity of numerical analyses accounting for material rate-sensitiveness.

In the last decades, several authors devoted both theoretical and numerical studies to investigate instabilities in rate-sensitive materials, such as metals [27, 28] and soils [23, 29, 30]. Nevertheless, a general framework establishing when viscoplastic constitutive instabilities can occur under mixed stress-strain loading programs $[31,32]$ is still missing in literature. These are actually very relevant in practice, because the loading processes in both experimental tests and real boundary value problems are usually characterized by a prescribed combination of certain stress and strain components.

This paper provides a sufficient condition for small-strain mechanical stability by explicitly taking into account the time-dependent response of geomaterials. As will be further clarified, the proposed theoretical framework exhibits strict connections to the elasto-plastic 'theory of controllability', first proposed and developed by Nova and coworkers [31-33]. It will be shown that, despite the different mathematical structures of constitutive equations, the results from the rate-dependent and inviscid theories are closely connected.

\section{Notation}

For analytical convenience, a matrix notation is hereafter adopted. Column vectors and square matrices are used to represent second-order and fourth-order tensors, respectively. Vectors and matrices are denoted by bold symbols, while the superscript ${ }^{T}$ stands for transposition. The partial derivative operator is $\partial / \partial$, whereas total derivatives are meant by $d / d$. Dots and double dots are also employed for first and second total time derivatives. Henceforth, $t$ will be standing for physical time.

\section{LOSS OF STABILITY/CONTROLLABILITY IN INVISCID ELASTO-PLASTIC SOLIDS}

Some relevant concepts about the loss of stability and controllability in rate-insensitive solids are hereafter summarized. While in this section only essential details for following developments are recalled, interested readers can find in the wide works by [34-37] in-depth explanations (and more references) about stability issues in elasto-plastic continua.

In the context of single potential elasto-platicity, incremental non-linearity is lumped into the two options of elasto-plastic loading and elastic unloading (only two tensorial stress zones exist $[38,39])$, so that stability analyses are meaningful in the inelastic regime exclusively.

It is first important to recall the well-known stability criterion proposed by [40], stating that the material response is stable as long as the second-order work density $d^{2} W$ is positive under any incremental perturbation [36]:

$$
d^{2} W=\frac{1}{2} d \sigma^{T} d \varepsilon>0 \quad \forall \quad d \varepsilon
$$


in which $d \boldsymbol{\sigma}$ and $d \boldsymbol{\varepsilon}$ are the incremental stress and strain (column) vectors. The Hill's sufficient stability condition was then applied by [41] to highlight the implications of non-associated plastic flow rules.

Years later, [33] further pointed out the meaning of the analysis by [41] in the light of the 'theory of controllability' introduced by Nova and coworkers [31,32]. In fact, it is possible to demonstrate that the admissibility of the elastic-plastic response depends on the current hardening modulus $H$ and the hardening limits $H_{1}$ and $H_{2}$ defined by [41]:

1. If $H>H_{1}$, then existence and uniqueness are guaranteed for any incremental loading path and any loading control (unconditional stability);

2. If $H_{2} \leq H \leq H_{1}$, then existence and uniqueness of the incremental response are a function of the loading program (conditional stability);

3. If $\mathrm{H}<\mathrm{H}_{2}$, then either the incremental response does not exist or the solution is not unique (unconditional instability).

Reference [33] drew these conclusions by analyzing the incremental elastic-plastic response under mixed loading conditions, that is, by assuming that certain stress and strain components $\sigma_{\alpha}$ and $\boldsymbol{\varepsilon}_{\beta}$ are controlled during the loading process. In general, any mixed loading control can be formulated by defining in $I=\{i \in \mathbb{N}: i=1,2, \ldots, 6\}$ two subsets $\alpha$ and $\beta$ containing the row indexes of the controlled stress and strain components, respectively:

$$
\begin{aligned}
\alpha & \subseteq I, \beta \subseteq I \\
\alpha \cap \beta & =\varnothing, \alpha \cup \beta=I \Rightarrow|\alpha|+|\beta|=|I|=6
\end{aligned}
$$

where $\mid$ denotes the set cardinality (number of elements in the set). Compatible $\alpha$ and $\beta$ are, for instance, $\alpha=\{1,3,5\}$ and $\beta=\{2,4,6\}$, or $\alpha=\{2,3\}$ and $\beta=\{1,4,5,6\}$, and so on. Mixed loading programs spontaneously induce a rearrangement of the incremental constitutive relationship, separating known and unknown stress/strain components:

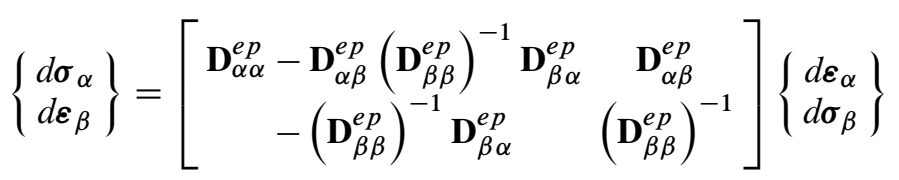

where $\mathbf{D}^{e p}$ the tangent elasto-plastic stiffness matrix (inverse of the compliance matrix $\mathbf{C}^{e p}$ ).

The partitioned matrix form (3) is meaningful only on condition that the non-negativeness of the plastic multiplier $\Lambda$ is ensured, whose general expression for mixed loading programs is [33]:

$$
\Lambda=\frac{1}{H-H_{\chi}} \frac{\partial f^{T}}{\partial \boldsymbol{\sigma}} d \boldsymbol{\sigma}^{t r}, \quad H=-\frac{\partial f^{T}}{\partial \boldsymbol{q}} \frac{\partial \boldsymbol{q}}{\partial \boldsymbol{\varepsilon} p} \frac{\partial g}{\partial \boldsymbol{\sigma}}
$$

where (i) the yield function $f$ depends on $\boldsymbol{\sigma}$ and a vector $\boldsymbol{q}$ of hardening variables; (ii) the gradient of the plastic potential $g$ identifies the direction of the plastic strain increment; and (iii) the so-called incremental trial stress $d \sigma^{t r}$ is a function of the prescribed stress/strain increments $\left(d \sigma_{\alpha}\right.$ and $\left.d \varepsilon_{\beta}\right)$ and of certain sub-blocks of the elastic stiffness/compliance matrices $\mathbf{D}^{e l} / \mathbf{C}^{e l}$ [33].

Equation (4) points out that the plastic multiplier tends to infinity as the hardening modulus $H$ approaches the so-called 'modulus of controllability' $H_{\chi}$ :

$$
\begin{aligned}
H_{\chi} & =-\frac{\partial f^{T}}{\partial \sigma_{\beta}}\left[\mathbf{D}_{\beta \beta}^{e l}-\mathbf{D}_{\beta \alpha}^{e l}\left(\mathbf{D}_{\alpha \alpha}^{e l}\right)^{-1} \mathbf{D}_{\alpha \beta}^{e l}\right] \frac{\partial g}{\partial \sigma_{\beta}}= \\
& =-\frac{\partial f^{T}}{\partial \sigma_{\beta}}\left(\mathbf{C}_{\beta \beta}^{e l}\right)^{-1} \frac{\partial g^{T}}{\partial \sigma_{\beta}}
\end{aligned}
$$

The definition $H_{\chi}$ depends on the actual loading constraints through $\alpha$ and $\beta$ in (2) and suggests the formulation of the following stability/controllability criterion [33]:

$$
H>H_{\chi}
$$


ensuring the positiveness of the plastic multiplier in (4). Incidentally, it could be demonstrated that $H_{\chi}$ is always in the range bounded by $H_{1}$ and $H_{2}$ as defined by [41].

Condition (6) can be specialized for the cases of pure stress and strain control [41]:

$$
\begin{gathered}
\text { stress control: } \quad \alpha=I, \beta=\varnothing \Longrightarrow H>H_{\chi}=0 \\
\text { strain control: } \alpha=\varnothing, \beta=I \Longrightarrow H>H_{\chi}=H_{c}, \quad H_{c}=-\frac{\partial f^{T}}{\partial \boldsymbol{\sigma}} \mathbf{D}^{e l} \frac{\partial g}{\partial \boldsymbol{\sigma}}
\end{gathered}
$$

where $H_{c}$ is the so-called critical hardening modulus [42].

The earlier approach is in essence very simple and flexible, but not employable for elastoviscoplastic solids. Indeed, the incremental form (3) with tangent stiffness/compliance matrices can never be retrieved for elasto-viscoplastic constitutive relationships [43].

\section{PERZYNA'S THEORY FOR RATE-DEPENDENT PLASTICITY}

\subsection{General concepts}

The theory of elasto-viscoplasticity relies on the assumption that the reversible (elastic) and the unrecoverable (viscoplastic) components of the total deformation combine additively. This implies that the total strain rate can be decomposed as

$$
\frac{d \boldsymbol{\varepsilon}}{d t}=\frac{d \boldsymbol{\varepsilon}^{e l}}{d t}+\frac{d \boldsymbol{\varepsilon}^{v p}}{d t}
$$

where the superscripts $e l$ and $v p$ stand for elastic and viscoplastic, respectively. The simplest assumption of isotropic linear response is here introduced for the elastic deformation:

$$
\frac{d \boldsymbol{\varepsilon}^{e l}}{d t}=\mathbf{C}^{e l} \frac{d \boldsymbol{\sigma}}{d t} \quad \text { or } \quad \frac{d \boldsymbol{\sigma}}{d t}=\mathbf{D}^{e l} \frac{d \boldsymbol{\varepsilon}^{e l}}{d t}
$$

while the viscoplastic strain rate is here obtained through the well-known Perzyna's approach $[13,14]$ :

$$
\frac{d \boldsymbol{\varepsilon}^{v p}}{d t}=\Phi(f) \frac{\partial g}{\partial \boldsymbol{\sigma}}
$$

According to Equation (11), the scalar $\Phi$ function (the so-called 'viscous nucleus') has a major influence on the magnitude of the viscoplastic strain rate ${ }^{\ddagger}$, while its direction in the strain rate space is given by the stress gradient of the plastic potential $g$. The enforcement of the plastic consistency condition is unnecessary, because the time rate of $\boldsymbol{\varepsilon}^{v p}$ is directly derived from $\Phi$. As a consequence, when plastifications take place, the stress state is not constrained to lie on the yield locus $f=0$ and 'overstresses' occur.

In most cases, the viscous nucleus $\Phi$ is a non-negative non-decreasing function of the yield function $f(\Phi \geq 0$ and $d \Phi / d f \geq 0)$ [19]. Provided the analytical definitions of the yield locus, the plastic potential and the hardening rules, a constitutive model can be easily formulated as either elasto-plastic or elasto-viscoplastic depending on the flow rule adopted. In this respect, the following property holds [19]:

$$
\{\Phi \geq 0 \quad \forall f \geq 0 ; \quad \Phi=0 \quad \forall f<0\} \Longrightarrow \int_{0}^{+\infty} \frac{d \boldsymbol{\varepsilon}^{v p}}{d t} d t=d \boldsymbol{\varepsilon}^{p}
$$

where $d \boldsymbol{\varepsilon}^{p}$ is the plastic strain increment produced by the corresponding inviscid flow rule. In other words, statement (12) implies that, as long as no viscoplastic strains develop when $f<0$, the

\footnotetext{
${ }^{\ddagger}$ Strictly speaking, there is also an influence of the plastic potential gradient. This could be easily eliminated by introducing $\frac{\partial g}{\partial \boldsymbol{\sigma}} /\left|\frac{\partial g}{\partial \boldsymbol{\sigma}}\right|$ in Equation (11).
} 
viscoplastic strain increment induced by a given perturbation tends, after an infinite amount of time, to the instantaneous plastic response: from this standpoint, standard plasticity can be regarded as the limit of viscoplasticity at vanishing rate-sensitiveness (or infinitely slow loading). It could be also proven that, as the elasto-plastic limit is approached, $\Phi \rightarrow \infty$ (infinite plastic strain rate) and $f \rightarrow 0$ (consistency satisfied).

\subsection{Second-order form of constitutive equations}

As a preliminary step, the following stability analysis requires a second-order form of Perzyna constitutive equations to be derived by time differentiation for mixed loading programs.

For this purpose, the authors assumed that (i) $d \mathbf{D}^{e l} / d t=d \mathbf{C}^{e l} / d t=\mathbf{0}$ (constant elastic parameters) and (ii) the yield function $f$ and the plastic potential $g$ depend on time only through the stress vector $\boldsymbol{\sigma}$ and the vectors of hardening variables $\boldsymbol{q}$ and $\boldsymbol{p}$ :

$$
\begin{aligned}
& f(t)=f\left\{\boldsymbol{\sigma}(t), \boldsymbol{q}\left[\boldsymbol{\varepsilon}^{v p}(t)\right]\right\} \\
& g(t)=g\left\{\boldsymbol{\sigma}(t), \boldsymbol{p}\left[\boldsymbol{\varepsilon}^{v p}(t)\right]\right\}
\end{aligned}
$$

Relationships (13) and (14) come from the assumption of strain-hardening material, whereas no dependence of the hardening variables on the inelastic strain rate is considered [15, 23, 29]. Accordingly, the second time derivative of the viscoplastic strain rate can be easily obtained by deriving the Perzyna's flow rule (11):

$$
\begin{aligned}
\frac{d^{2} \boldsymbol{\varepsilon}^{v p}}{d t^{2}} & =\frac{d \Phi}{d t} \frac{\partial g}{\partial \boldsymbol{\sigma}}+\Phi \frac{d}{d t}\left(\frac{\partial g}{\partial \boldsymbol{\sigma}}\right)= \\
& =\frac{d \Phi}{d f}\left(\frac{\partial f^{T}}{\partial \boldsymbol{\sigma}} \frac{d \boldsymbol{\sigma}}{d t}-\Phi H\right) \frac{\partial g}{\partial \boldsymbol{\sigma}}+\Phi\left(\frac{\partial^{2} g}{\partial \boldsymbol{\sigma} \otimes \partial \boldsymbol{\sigma}} \frac{d \boldsymbol{\sigma}}{d t}+\frac{\partial^{2} g}{\partial \boldsymbol{\sigma} \otimes \partial \boldsymbol{p}} \frac{\partial \boldsymbol{p}}{\partial \boldsymbol{\varepsilon}^{v p}} \frac{d \boldsymbol{\varepsilon}^{v p}}{d t}\right)
\end{aligned}
$$

in which the viscous nucleus $\Phi$ and its $f$-derivative, the hardening modulus $H$ and the derivatives of $g$ with respect to $\boldsymbol{\sigma}$ and $\boldsymbol{p}$ appear.

For the sake of clarity, the time derivation of constitutive equations is first performed for the simpler cases of full stress and strain control; then, the general mixed loading case is addressed. The most cumbersome analytical developments are skipped here and summarized in Appendix 5.

3.2.1. Stress control $(\alpha=I, \beta=\varnothing)$. Under full stress control, a stress vector time history $\Sigma(t)$ is prescribed:

$$
\boldsymbol{\sigma}(t)=\boldsymbol{\Sigma}(t) \Rightarrow \frac{d \boldsymbol{\varepsilon}^{v p}}{d t}=\frac{d \boldsymbol{\varepsilon}}{d t}-\mathbf{C}^{e l} \dot{\boldsymbol{\Sigma}}
$$

so that the second time derivative of the (unknown) strain vector can be expressed as

$$
\frac{d^{2} \boldsymbol{\varepsilon}}{d t^{2}}=\frac{d^{2} \boldsymbol{\varepsilon}^{e l}}{d t^{2}}+\frac{d^{2} \boldsymbol{\varepsilon}^{v p}}{d t^{2}}=\mathbf{C}^{e l} \ddot{\boldsymbol{\Sigma}}+\frac{d^{2} \boldsymbol{\varepsilon}^{v p}}{d t^{2}}
$$

By combining the strain splitting (17) with the stress control constraint (16) and the second-order flow rule (15), the following $\mathrm{ODE}^{\S}$ system is retrieved (see Appendix 5):

$$
\frac{d \mathbf{X}}{d t}=\mathbf{A}_{\sigma} \mathbf{X}+\mathbf{F}_{\sigma}
$$

in which $\mathbf{X}=\frac{d \boldsymbol{\varepsilon}}{d t}$ has been set and

$$
\mathbf{A}_{\sigma}=-\frac{d \Phi}{d f}\left(H+\frac{H_{\dot{\Sigma}}}{\Phi}\right) \mathbf{I}_{I}+\Phi \frac{\partial^{2} g}{\partial \boldsymbol{\sigma} \otimes \partial \boldsymbol{p}} \frac{\partial \boldsymbol{p}}{\partial \boldsymbol{\varepsilon}^{v p}}
$$

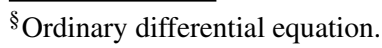




$$
\begin{gathered}
\mathbf{F}_{\sigma}=\mathbf{C}^{e l} \ddot{\boldsymbol{\Sigma}}+\Phi\left(\frac{\partial^{2} g}{\partial \boldsymbol{\sigma} \otimes \partial \boldsymbol{\sigma}}-\frac{\partial^{2} g}{\partial \boldsymbol{\sigma} \otimes \partial \boldsymbol{p}} \frac{\partial \boldsymbol{p}}{\partial \boldsymbol{\varepsilon}^{v p}} \mathbf{C}^{e l}\right) \dot{\boldsymbol{\Sigma}}+\frac{d \Phi}{d f}\left(H+\frac{H_{\dot{\Sigma}}}{\Phi}\right) \mathbf{C}^{e l} \dot{\boldsymbol{\Sigma}} \\
H_{\dot{\Sigma}}=-\frac{\partial f^{T}}{\partial \boldsymbol{\sigma}} \dot{\boldsymbol{\Sigma}}
\end{gathered}
$$

$\mathbf{I}_{I}$ stands for a $6 \times 6$-sized identity matrix (its size coincides with the cardinality of the set $I$ ).

The linear system (18) governs the strain acceleration (rate of the total strain rate) under a prescribed stress history. It is also worth observing that:

- since the entries of the matrix $\mathbf{A}_{\sigma}$ evolve with the stress-strain state, system (18) is time-varying (non-stationary);

- the vector $\mathbf{F}_{\sigma}$ vanishes for creep loading conditions (i.e., under constant stress and $\dot{\boldsymbol{\Sigma}}=\ddot{\boldsymbol{\Sigma}}=$ 0), and so does the scalar $H_{\dot{\Sigma}}$ in (21) - which will be henceforth referred to as 'stress rate modulus'.

3.2.2. Strain control $(\alpha=\varnothing, \beta=I)$. In case the loading program is fully strain-controlled, the total strain vector time history $\boldsymbol{E}(t)$ is prescribed:

$$
\boldsymbol{\varepsilon}(t)=\boldsymbol{E}(t) \Rightarrow \frac{d \boldsymbol{\varepsilon}^{v p}}{d t}=\dot{\boldsymbol{E}}-\mathbf{C}^{e l} \frac{d \boldsymbol{\sigma}}{d t}
$$

and the onset of positive accelerations can be detected by monitoring the second time derivative of the (unknown) stress vector:

$$
\frac{d^{2} \sigma}{d t^{2}}=\mathbf{D}^{e l}\left(\frac{d^{2} \boldsymbol{\varepsilon}}{d t^{2}}-\frac{d^{2} \boldsymbol{\varepsilon}^{v p}}{d t^{2}}\right)=\mathbf{D}^{e l} \ddot{\boldsymbol{E}}-\mathbf{D}^{e l} \frac{d^{2} \boldsymbol{\varepsilon}^{v p}}{d t^{2}}
$$

After some derivations similar to those performed for the stress-controlled case (Appendix 5), the following ODE system is found:

$$
\frac{d \mathbf{X}}{d t}=\mathbf{A}_{\varepsilon} \mathbf{X}+\mathbf{F}_{\varepsilon}
$$

where $\mathbf{X}=d \boldsymbol{\sigma} / d t$ and

$$
\begin{gathered}
\mathbf{A}_{\varepsilon}=\left[-\frac{d \Phi}{d f}\left(H-H_{c}+\frac{H_{\dot{E}}}{\Phi}\right) \mathbf{I}_{I}-\Phi \mathbf{D}^{e l}\left(\frac{\partial^{2} g}{\partial \boldsymbol{\sigma} \otimes \partial \boldsymbol{\sigma}}-\frac{\partial^{2} g}{\partial \boldsymbol{\sigma} \otimes \partial \boldsymbol{p}} \frac{\partial \boldsymbol{p}}{\partial \boldsymbol{\varepsilon}^{v p}} \mathbf{C}^{e l}\right)\right] \\
\mathbf{F}_{\varepsilon}=\mathbf{D}^{e l} \ddot{\boldsymbol{E}}+\frac{d \Phi}{d f}\left(H-H_{c}+\frac{H_{\dot{E}}}{\Phi}\right) \mathbf{D}^{e l} \dot{\boldsymbol{E}}-\Phi \mathbf{D}^{e l} \frac{\partial^{2} g}{\partial \boldsymbol{\sigma} \otimes \partial \boldsymbol{\beta}} \frac{\partial \boldsymbol{\beta}}{\partial \boldsymbol{\varepsilon}^{v p}} \dot{\boldsymbol{E}}= \\
=\mathbf{D}^{e l} \ddot{\boldsymbol{E}}+\mathbf{D}^{e l}\left[\frac{d \Phi}{d f}\left(H-H_{c}+\frac{H_{\dot{E}}}{\Phi}\right) \mathbf{I}_{I}-\Phi \frac{\partial^{2} g}{\partial \boldsymbol{\sigma} \otimes \partial \boldsymbol{p}} \frac{\partial \boldsymbol{p}}{\boldsymbol{\varepsilon}^{v p}}\right] \dot{\boldsymbol{E}} \\
H_{\dot{E}}=-\frac{\partial f^{T}}{\partial \boldsymbol{\sigma}} \mathbf{D}^{e l} \dot{\boldsymbol{E}}
\end{gathered}
$$

Apparently, systems (18) and (24) possess the same mathematical structure. In the latter, the assumption of prescribed strain vector leads to retrieve the critical softening modulus $H_{c}$ in Equation 8 [41], along with the newly defined 'strain rate modulus' $H_{\dot{E}}$ in (27). 
3.2.3. Mixed stress-strain control. Under general mixed loading, a combination of certain stress $\left(\sigma_{\alpha}\right)$ and strain $\left(\varepsilon_{\beta}\right)$ components is known/controlled. By following the approach recalled in Section 2 , the total strain vector and its elastic and viscoplastic components can be partitioned as follows ${ }^{\mathrm{II}}$ :

$$
\frac{d}{d t}\left\{\begin{array}{c}
\boldsymbol{\varepsilon}_{\alpha} \\
\boldsymbol{\varepsilon}_{\beta}
\end{array}\right\}=\left[\begin{array}{ll}
\mathbf{C}_{\alpha \alpha} & \mathbf{C}_{\alpha \beta} \\
\mathbf{C}_{\beta \alpha} & \mathbf{C}_{\beta \beta}
\end{array}\right] \frac{d}{d t}\left\{\begin{array}{l}
\sigma_{\alpha} \\
\sigma_{\beta}
\end{array}\right\}+\Phi\left\{\begin{array}{c}
\frac{\partial g}{\partial \sigma_{\alpha}} \\
\frac{\partial g}{\partial \sigma_{\beta}}
\end{array}\right\}
$$

Hereafter, the constraints on the prescribed stress and strain components are given to define the loading program:

$$
\sigma_{\alpha}(t)=\Sigma_{\alpha}(t) \quad \varepsilon_{\beta}(t)=E_{\beta}(t)
$$

whence the following relationships for the viscoplastic strain rates result:

$$
\begin{aligned}
& \frac{d \boldsymbol{\varepsilon}_{\alpha}^{v p}}{d t}=\frac{d \boldsymbol{\varepsilon}_{\alpha}}{d t}-\mathbf{C}_{\alpha \alpha} \frac{d \boldsymbol{\sigma}_{\alpha}}{d t}-\mathbf{C}_{\alpha \beta} \frac{d \boldsymbol{\sigma}_{\beta}}{d t}=\frac{d \boldsymbol{\varepsilon}_{\alpha}}{d t}-\mathbf{C}_{\alpha \alpha} \dot{\boldsymbol{\Sigma}}_{\alpha}-\mathbf{C}_{\alpha \beta} \frac{d \boldsymbol{\sigma}_{\beta}}{d t} \\
& \frac{d \boldsymbol{\varepsilon}_{\beta}^{v p}}{d t}=\frac{d \boldsymbol{\varepsilon}_{\beta}}{d t}-\mathbf{C}_{\beta \alpha} \frac{d \boldsymbol{\sigma}_{\alpha}}{d t}-\mathbf{C}_{\beta \beta} \frac{d \boldsymbol{\sigma}_{\beta}}{d t}=\dot{\boldsymbol{E}}_{\beta}-\mathbf{C}_{\beta \alpha} \dot{\boldsymbol{\Sigma}}_{\alpha}-\mathbf{C}_{\beta \beta} \frac{d \boldsymbol{\sigma}_{\beta}}{d t}
\end{aligned}
$$

The substitution of the loading constraints (29) into the constitutive law (28) generalizes the Perzyna's flow rule (11) in the sense of mixed loading programs. Accordingly, the rates of the uncontrolled stresses and strains assume the form

$$
\frac{d}{d t}\left\{\begin{array}{c}
\boldsymbol{\varepsilon}_{\alpha} \\
\boldsymbol{\sigma}_{\beta}
\end{array}\right\}=\Phi\left[\begin{array}{cc}
\mathbf{I}_{\alpha \alpha} & -\mathbf{C}_{\alpha \beta} \mathbf{C}_{\beta \beta}^{-1} \\
\mathbf{0} & -\mathbf{C}_{\beta \beta}^{-1}
\end{array}\right]\left\{\begin{array}{c}
\frac{\partial g}{\partial \boldsymbol{\sigma}_{\alpha}} \\
\frac{\partial g}{\partial \boldsymbol{\sigma}_{\beta}}
\end{array}\right\}+\left\{\begin{array}{c}
\dot{\boldsymbol{\Omega}}_{\alpha} \\
\dot{\boldsymbol{\Omega}}_{\beta}
\end{array}\right\}
$$

where the vector $\boldsymbol{\Omega}$

$$
\left\{\begin{array}{c}
\dot{\boldsymbol{\Omega}}_{\alpha} \\
\dot{\boldsymbol{\Omega}}_{\beta}
\end{array}\right\}=\left\{\begin{array}{c}
\mathbf{C}_{\alpha \alpha} \dot{\boldsymbol{\Sigma}}_{\alpha}+\mathbf{C}_{\alpha \beta} \mathbf{C}_{\beta \beta}^{-1}\left(\dot{\boldsymbol{E}}_{\beta}-\mathbf{C}_{\beta \alpha} \dot{\boldsymbol{\Sigma}}_{\alpha}\right) \\
\mathbf{C}_{\beta \beta}^{-1}\left(\dot{\boldsymbol{E}}_{\beta}-\mathbf{C}_{\beta \alpha} \dot{\boldsymbol{\Sigma}}_{\alpha}\right)
\end{array}\right\}
$$

vanishes at constant $\boldsymbol{\Sigma}_{\alpha}$ and $\boldsymbol{E}_{\beta}$. Then, both the equations in system (31) are further differentiated with respect to time:

$$
\begin{aligned}
\frac{d^{2} \boldsymbol{\varepsilon}_{\alpha}}{d t^{2}} & =\frac{d}{d t}\left(\Phi \frac{\partial g}{\partial \boldsymbol{\sigma}_{\alpha}}-\mathbf{C}_{\alpha \beta} \mathbf{C}_{\beta \beta}^{-1} \Phi \frac{\partial g}{\partial \boldsymbol{\sigma}_{\beta}}+\dot{\boldsymbol{\Omega}}_{\alpha}\right)= \\
& =\frac{d \Phi}{d t} \frac{\partial g}{\partial \boldsymbol{\sigma}_{\alpha}}+\Phi \frac{d}{d t}\left(\frac{\partial g}{\partial \boldsymbol{\sigma}_{\alpha}}\right)-\frac{d \Phi}{d t} \mathbf{C}_{\alpha \beta} \mathbf{C}_{\beta \beta}^{-1} \frac{\partial g}{\partial \boldsymbol{\sigma}_{\beta}}-\Phi \mathbf{C}_{\alpha \beta} \mathbf{C}_{\beta \beta}^{-1} \frac{d}{d t}\left(\frac{\partial g}{\boldsymbol{\sigma}_{\beta}}\right)+\ddot{\boldsymbol{\Omega}}_{\alpha} \\
\frac{d^{2} \boldsymbol{\sigma}_{\beta}}{d t^{2}} & =\frac{d}{d t}\left(-\mathbf{C}_{\beta \beta}^{-1} \Phi \frac{\partial g}{\partial \boldsymbol{\sigma}_{\beta}}+\dot{\boldsymbol{\Omega}}_{\beta}\right)=-\frac{d \Phi}{d t} \mathbf{C}_{\beta \beta}^{-1} \frac{\partial g}{\partial \boldsymbol{\sigma}_{\beta}}-\Phi \mathbf{C}_{\beta \beta}^{-1} \frac{d}{d t}\left(\frac{\partial g}{\partial \boldsymbol{\sigma}_{\beta}}\right)+\ddot{\boldsymbol{\Omega}}_{\beta}
\end{aligned}
$$

Even in this case, some more demanding manipulations (5) enable to recast Equations 33 and (34) as a time-varying ODE system:

$$
\frac{d \mathbf{X}}{d t}=\mathbf{A} \mathbf{X}+\mathbf{F} \Rightarrow \frac{d^{2}}{d t^{2}}\left\{\begin{array}{l}
\boldsymbol{\varepsilon}_{\alpha} \\
\boldsymbol{\sigma}_{\beta}
\end{array}\right\}=\left[\begin{array}{ll}
\mathbf{A}_{\alpha \alpha} & \mathbf{A}_{\alpha \beta} \\
\mathbf{A}_{\beta \alpha} & \mathbf{A}_{\beta \beta}
\end{array}\right] \frac{d}{d t}\left\{\begin{array}{l}
\boldsymbol{\varepsilon}_{\alpha} \\
\boldsymbol{\sigma}_{\beta}
\end{array}\right\}+\left\{\begin{array}{l}
\mathbf{F}_{\alpha} \\
\mathbf{F}_{\beta}
\end{array}\right\}
$$

For the sake of analytical convenience, the sub-blocks in (35) are now specified for the special case:

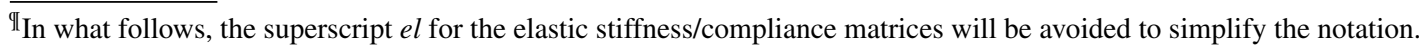




$$
\frac{\partial}{\partial \boldsymbol{p}}\left(\frac{\partial g}{\partial \boldsymbol{\sigma}}\right)=\frac{\partial^{2} g}{\partial \boldsymbol{\sigma} \otimes \partial \boldsymbol{p}}=\mathbf{0}
$$

meaning no influence of the $g$-hardening variables on the direction of the viscoplastic flow (Equation 11). This assumption implies some loss of generality but still allows to address relevant cases, including perfectly viscoplastic (no hardening) and Cam-Clay-type (isotropic strainhardening) constitutive relationships. In particular, the latter are widely used to model the non-linear behavior of soils under monotonic loading (see e.g. [44] for details).

The simplification (36) leads to the following sub-block expressions:

$$
\begin{gathered}
\mathbf{A}_{\alpha \alpha}=-\frac{d \Phi}{d f}\left(H-H_{\chi}+\frac{H_{\dot{\Sigma} \dot{E}}}{\Phi}\right) \mathbf{I}_{\alpha \alpha} \\
\mathbf{A}_{\alpha \beta}=\Phi\left(\frac{\partial^{2} g}{\partial \boldsymbol{\sigma}_{\alpha} \otimes \partial \sigma_{\beta}}-\mathbf{C}_{\alpha \beta} \mathbf{C}_{\beta \beta}^{-1} \frac{\partial^{2} g}{\partial \sigma_{\beta} \otimes \partial \boldsymbol{\sigma}_{\beta}}\right) \\
\mathbf{A}_{\beta \alpha}=\mathbf{0} \\
\mathbf{A}_{\beta \beta}=-\frac{d \Phi}{d f}\left(H-H_{\chi}+\frac{H_{\dot{\Sigma} \dot{E}}}{\Phi}\right) \mathbf{I}_{\beta \beta}-\Phi \mathbf{C}_{\beta \beta}^{-1} \frac{\partial^{2} g}{\partial \boldsymbol{\sigma}_{\beta} \otimes \partial \boldsymbol{\sigma}_{\beta}}
\end{gathered}
$$

and

$$
\begin{gathered}
\mathbf{F}_{\alpha}=\ddot{\boldsymbol{\Omega}}_{\alpha}+\frac{d \Phi}{d f}\left(H-H_{\chi}+\frac{H_{\dot{\Sigma} \dot{E}}}{\Phi}\right)\left[\mathbf{C}_{\alpha \alpha} \dot{\boldsymbol{\Sigma}}_{\alpha}-\mathbf{C}_{\alpha \beta} \mathbf{C}_{\beta \beta}^{-1}\left(\mathbf{C}_{\beta \alpha} \dot{\boldsymbol{\Sigma}}_{\alpha}-\dot{\boldsymbol{E}}_{\beta}\right)\right]+ \\
+\Phi\left(\frac{\partial^{2} g}{\partial \boldsymbol{\sigma}_{\alpha} \otimes \partial \sigma_{\alpha}}-\mathbf{C}_{\alpha \beta} \mathbf{C}_{\beta \beta}^{-1} \frac{\partial^{2} g}{\partial \boldsymbol{\sigma}_{\beta} \otimes \partial \boldsymbol{\sigma}_{\alpha}}\right) \dot{\boldsymbol{\Sigma}}_{\alpha} \\
\mathbf{F}_{\beta}=\ddot{\boldsymbol{\Omega}}_{\beta}-\frac{d \Phi}{d f}\left(H-H_{\chi}+\frac{H_{\dot{\Sigma} \dot{\Sigma}}}{\Phi}\right) \mathbf{C}_{\beta \beta}^{-1}\left(\mathbf{C}_{\beta \alpha} \dot{\boldsymbol{\Sigma}}_{\alpha}-\dot{\boldsymbol{E}}_{\beta}\right)+ \\
-\Phi \mathbf{C}_{\beta \beta}^{-1} \frac{\partial^{2} g}{\partial \sigma_{\beta} \otimes \partial \sigma_{\alpha}} \dot{\boldsymbol{\Sigma}}_{\alpha}
\end{gathered}
$$

where

$$
H_{\dot{\Sigma} \dot{E}}=-\left(\frac{\partial f^{T}}{\partial \sigma_{\alpha}} \dot{\boldsymbol{\Sigma}}_{\alpha}+\frac{\partial f^{T}}{\partial \sigma_{\beta}} \dot{\boldsymbol{\Omega}}_{\beta}\right)
$$

has been posed. From the aforementioned relationships, it is possible to infer that:

- as in the elasto-plastic case, the response to mixed loading programs is influenced by the controllability modulus $H_{\chi}$ introduced by [33] (see in (5));

- the definition (43) of the so-called 'stress/strain rate modulus' $H_{\dot{\Sigma} \dot{E}}$ spontaneously arises as a generalization of $H_{\dot{\Sigma}}$ and $H_{\dot{E}} \cdot H_{\dot{\Sigma} \dot{E}}$ vanishes when the prescribed rates $\dot{\boldsymbol{\Sigma}}_{\alpha}$ and $\dot{\boldsymbol{E}}_{\beta}$ are nil;

- the nullity of the sub-block $\mathbf{A}_{\beta \alpha}$ is not a consequence of the simplifying assumption (36), but it stems from the general structure of system (31). In other words, the properties exhibited by $\mathbf{A}$ because of $\mathbf{A}_{\beta \alpha}=\mathbf{0}$ would keep holding also for hardening models with non-isotropic/homothetic strain-hardening. 


\section{ANALYSIS OF ELASTO-VISCOPLASTIC CONSTITUTIVE STABILITY}

Because Perzyna-type relationships cannot be written in the incremental form (3) [43], the elasto-plastic approach in Section 2 for the analysis of material stability is not suitable for viscoplastic continua. Conversely, the aforementioned second-order Perzyna equations can be fruitfully exploited to the same purpose.

The second-order Perzyna relationship (35) is in the form of a linear ODE system:

$$
\frac{d \mathbf{X}}{d t}=\mathbf{A} \mathbf{X}+\mathbf{F}
$$

On condition that $\mathbf{F}(t)=\mathbf{0}$, stationary motion conditions $(d \mathbf{X} / d t=\mathbf{0})$ are attained when $\mathbf{X}(t)=$ $\mathbf{0}$, which is thus referred to as 'equilibrium trajectory'. In the present context, $\mathbf{X}$ is composed of stress/strain rate components and the equilibrium $\mathbf{X}=\mathbf{0}$ actually denotes a quasi-static evolution of the constitutive response (i.e., at negligible stress/strain time rates).

According to the well-known Lyapunov's definition [36, 45, 46], the equilibrium trajectory $\mathbf{X}(t)=\mathbf{0}$ is stable over the time set $T=\left[t_{0},+\infty\right)$ if

$$
\begin{aligned}
& \forall t \in T, \quad \forall \varepsilon>0 \quad \exists \delta(\varepsilon)>0 \\
& \left\|\mathbf{X}\left(t_{0}\right)\right\|<\delta \Longrightarrow\|\mathbf{X}(t)\|<\varepsilon
\end{aligned}
$$

Roughly speaking, the stationary trajectory is said to be stable if other 'close' trajectories at a given initial time keep 'staying close' to it as time elapses. Posing $\mathbf{F}=\mathbf{0}$ to analyze the stability of the trajectory $\mathbf{X}(t)=\mathbf{0}$ has a clear physical motivation: stability is in fact an intrinsic property of the system under free motion conditions, whereas instability can be triggered any time by enforcing appropriate perturbations.

In the case of linear ODE systems, Lyapunov's theory of stability $[45,46]$ establishes a direct link between the spectral properties of the system matrix $\mathbf{A}$ and the stability of the equilibrium solution. In particular, it can be proven that:

1. $\mathbf{X}=\mathbf{0}$ is a stable equilibrium in the sense of (44) if the real parts of all the eigenvalues in $\Lambda$ (A) (spectrum of $\mathbf{A})$ are non-positive.

However, because matrix $\mathbf{A}$ is actually time-varying in the viscoplastic regime, the previous stability criterion is sufficient for stability, not necessary: in fact, if positive eigenvalues arise at some time $t$, the subsequent evolution of the system can be either stable or unstable depending on the actual $\mathbf{A}$ entries at elapsing time. It is only ensured that instability cannot occur while $\Lambda(\mathbf{A})$ is all formed by non-positive eigenvalues at each time $t$.

As is discussed in 5, all the eigenvalues in $\Lambda$ (A) for $\dot{\boldsymbol{\Sigma}}_{\alpha}=\mathbf{0}$ and $\dot{\boldsymbol{E}}_{\beta}=\mathbf{0}$ (nil external loading rates in (35)) are real and semisimple under very reasonable assumptions. As a consequence, the fulfillment of the sufficient stability condition (i.e., non-positive eigenvalues) also implies that $\mathbf{A}$ is negative semi-definite:

$$
\begin{aligned}
\lambda^{i} \leq 0 & \forall \lambda^{i} \in \Lambda(\mathbf{A}) \\
\Longrightarrow \mathbf{X}^{T} \mathbf{A X} \leq 0 & \forall \mathbf{X} \neq \mathbf{0} \\
\Longrightarrow \mathbf{X}^{T} \frac{d \mathbf{X}}{d t} \leq 0 & \forall \mathbf{X} \neq \mathbf{0} \wedge \mathbf{F}=\mathbf{0}
\end{aligned}
$$

whose mechanical interpretation is given in the next section.

\subsection{Mechanical interpretation}

Corollary (45) enables an enlightening mechanical/geometrical interpretation, which can be easily illustrated in the case of stress-controlled conditions (creep tests). Figure 1 qualitatively depicts in the strain rate space a situation in which the stability of the constitutive response is no longer ensured. At time $t$, an instantaneous increase in the strain rate norm is produced by a positive strain 


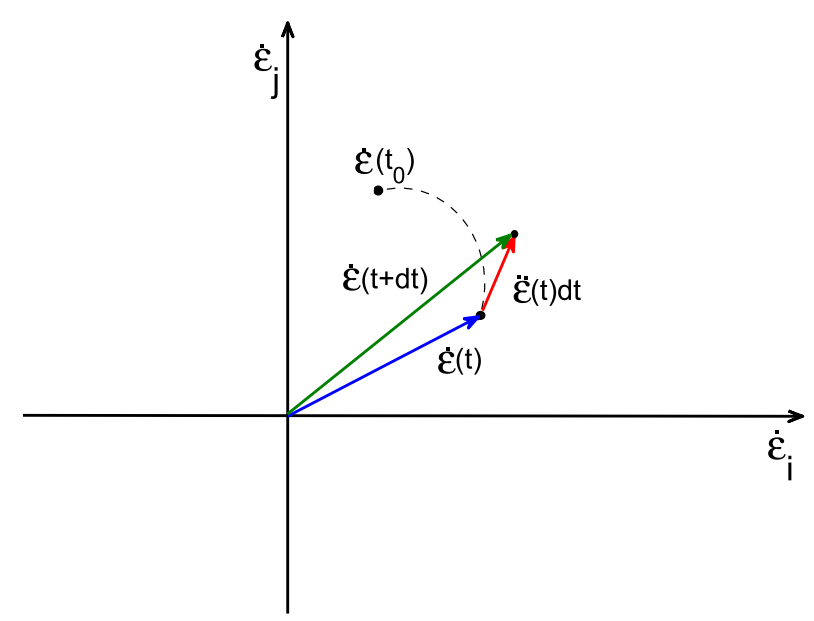

Figure 1. Representation of strain acceleration in the strain rate space.

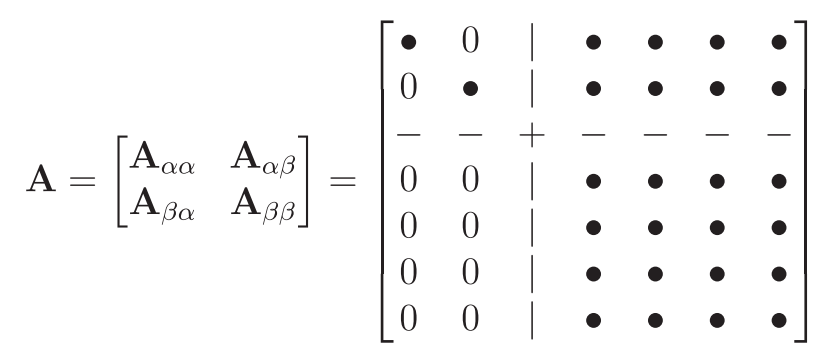

Figure 2. General structure of the partitioned matrix $\mathbf{A}$.

acceleration and a necessary step towards instability is taken. Apparently, this can never happen while $\dot{\boldsymbol{\varepsilon}}$ and $\ddot{\boldsymbol{\varepsilon}}$ are orthogonal (constant $\dot{\boldsymbol{\varepsilon}}$ norm) or the angle in between them is acute (decreasing $\dot{\boldsymbol{\varepsilon}}$ norm). This is in essence what corollary (45) states and clarifies the effect of acceleration terms on the onset of constitutive instabilities [21, 23, 29, 30].

For the sake of clarity, corollary (45) is rewritten with explicit reference to mixed loading variables:

$$
\frac{d}{d t}\left\{\begin{array}{c}
\varepsilon_{\alpha} \\
\sigma_{\beta}
\end{array}\right\}^{T} \cdot \frac{d^{2}}{d t^{2}}\left\{\begin{array}{c}
\varepsilon_{\alpha} \\
\sigma_{\beta}
\end{array}\right\}<0
$$

where $\sigma_{\beta}$ and $\varepsilon_{\alpha}$ are still the uncontrolled stress/strain components. As [31] proved for elasto-plastic problems, a condition similar to (46) can be also derived for loading programs in which workconjugate variables are defined as a linear combination of certain stress and strain components (for instance, volumetric and deviatoric stress/strain invariants under triaxial loading conditions).

\subsection{Determination of viscoplastic stability limits}

In the light of the aforementioned criterion, the viscoplastic stability analysis requires the whole spectrum $\Lambda(\mathbf{A})$ to be determined. For this purpose, Figure 2 illustrates the general structure of matrix $\mathbf{A}$ in terms of nil and non-nil entries (Equations (37)-(40)), implying that $\Lambda$ (A) can be obtained by combining the spectra $\Lambda\left(\mathbf{A}_{\alpha \alpha}\right)$ and $\Lambda\left(\mathbf{A}_{\beta \beta}\right)$ :

$$
\Lambda(\mathbf{A})=\Lambda\left(\mathbf{A}_{\alpha \alpha}\right) \cup \Lambda\left(\mathbf{A}_{\beta \beta}\right)
$$




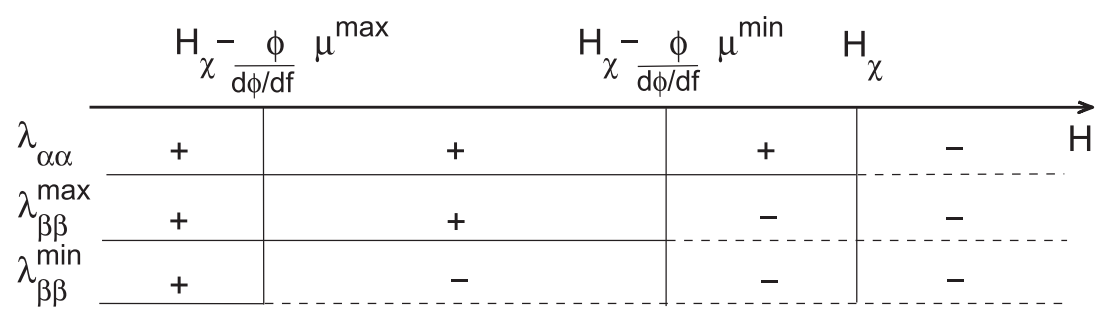

Figure 3. Sign of the eigenvalues in $\Lambda\left(\mathbf{A}_{\alpha \alpha}\right)$ and $\Lambda\left(\mathbf{A}_{\beta \beta}\right)$ as a function of the hardening modulus.

In particular, since $\mathbf{A}_{\alpha \alpha}$ is proportional to the identity matrix, each $i^{t h}$ eigenvalue $\lambda_{\alpha \alpha}^{i}$ in $\Lambda\left(\mathbf{A}_{\alpha \alpha}\right)$ assumes the following form for $\dot{\boldsymbol{\Sigma}}_{\alpha}=\mathbf{0}$ and $\dot{\boldsymbol{E}}_{\beta}=\mathbf{0}$ :

$$
\left.\lambda_{\alpha \alpha}^{i}\right|_{\dot{\boldsymbol{\Sigma}}_{\alpha}=\mathbf{0}, \dot{\boldsymbol{E}}_{\beta}=\mathbf{0}}=-\frac{d \Phi}{d f}\left(H-H_{\chi}\right) \quad \in \Lambda\left(\mathbf{A}_{\alpha \alpha}\right)
$$

Similarly, as $\mathbf{A}_{\beta \beta}$ is the sum of a diagonal matrix and a full matrix, it results:

$$
\left.\lambda_{\beta \beta}^{i}\right|_{\dot{\boldsymbol{\Sigma}}_{\alpha}=\mathbf{0}, \dot{\boldsymbol{E}}_{\beta}=\mathbf{0}}=-\frac{d \Phi}{d f}\left(H-H_{\chi}\right)-\Phi \mu^{i} \in \Lambda\left(\mathbf{A}_{\beta \beta}\right)
$$

where (Equation 40)

$$
\mu^{i} \in \Lambda(\mathbf{M}), \quad \mathbf{M}=\mathbf{C}_{\beta \beta}^{-1} \frac{\partial^{2} g}{\partial \boldsymbol{\sigma}_{\beta} \otimes \partial \boldsymbol{\sigma}_{\beta}}
$$

As is discussed in 5, the eigenvalues $\mu^{i}$ in $\mathbf{M}$ are all positive on condition that the plastic potential $g$ is convex in the stress space. If $\mu_{\max }$ is the maximum $\mu^{i}$, then only the following options are given (Figure 3):

1. $H \geq H_{\chi}$ : all the eigenvalues in $\Lambda(\mathbf{A})$ are non-positive and so does the quadratic form in (45) for any non-nil $\mathbf{X}$ (rate of the uncontrolled stress/strain components);

2. $H_{\chi}-\frac{\Phi}{d \Phi / d f} \mu_{\max }<H<H_{\chi}{ }^{\prime \prime}: \Lambda(\mathbf{A})$ is composed of both positive and negative eigenvalues, and the current sign of the quadratic form depends on the actual $\mathbf{X}$ value;

3. $H \leq H_{\chi}-\frac{\Phi}{d \Phi / d f} \mu_{\max }$ : all the eigenvalues in $\Lambda$ (A) are non-negative and the quadratic form is positive semi-definite.

As Figure 3 puts in evidence, the sufficient condition for viscoplastic stability is fulfilled as long as

$$
H>H_{\chi}
$$

This means that no viscoplastic constitutive instabilities can occur under mixed creep/relaxation programs while the hardening modulus $H$ is larger than the controllability modulus $H_{\chi}$ : the connection to the elasto-plastic condition given by [33] is self-evident. Importantly, option 2 testifies the existence of an $H$-range in which $\Lambda$ (A) includes both positive and negative eigenvalues and stability can no longer be ensured (either beneficial or detrimental accelerations will arise depending on the current $\mathbf{X}$ ). This also means that, in rate-sensitive solids, the state of the material is not only determined by 'static' variables (stresses, strains and hardening variables), but by their time rate as well.

\footnotetext{
"The properties of $\Phi$ recalled in Section 3.1 imply the positiveness of the ratio $\frac{\Phi}{d \Phi / d f}$.
} 
To further highlight the link between the viscoplastic and the elasto-plastic theories, it is worth showing what the viscous approach predicts at decreasing viscosity. For this purpose, a common analytical expression for $\Phi^{* *}$ is taken as a reference:

$$
\Phi(f)=\eta\langle f\rangle^{m}= \begin{cases}\eta f^{m} & f \geq 0 \\ 0 & f<0\end{cases}
$$

in which $\eta$ ('fluidity parameter') and $m$ are two constitutive parameters governing the material ratesensitivity. Although other options are available $([19,47])$, function (52) fulfills property (12) and the elasto-viscoplastic response tends to the elasto-plastic limit at vanishing rate-sensitiveness (i.e., at increasing $\eta$ and/or $m$ ). If, for example, $m=1$ is set in (52), then

$$
\left.\lambda_{\beta \beta}^{i}\right|_{\dot{\boldsymbol{\Sigma}}_{\alpha}=\mathbf{0}, \dot{\boldsymbol{E}}_{\beta}=\mathbf{0}}=-\eta\left[\left(H-H_{\chi}\right)+f \mu^{i}\right]
$$

and the inviscid limit reads:

$$
\begin{array}{r}
\eta \rightarrow \infty \Longrightarrow f \rightarrow 0 \\
\left.\lambda_{\beta \beta}^{i}\right|_{\dot{\boldsymbol{\Sigma}}_{\alpha}=\mathbf{0}, \dot{\boldsymbol{E}}_{\beta}=\mathbf{0}} ^{\eta \rightarrow \infty} \rightarrow-\eta\left(H-H_{\chi}\right)=\left.\lambda_{\alpha \alpha}^{i}\right|_{\dot{\boldsymbol{\Sigma}}_{\alpha}=\mathbf{0}, \dot{\boldsymbol{E}}_{\beta}=\mathbf{0}}
\end{array}
$$

Limit (54) shows that the eigenvalues in $\Lambda\left(\mathbf{A}_{\beta \beta}\right)$ and $\Lambda\left(\mathbf{A}_{\alpha \alpha}\right)$ tend to coincide as the fluidity parameter $\eta$ approaches infinity. This stems from the fact that, at vanishing rate-sensitiveness, the constitutive equations produce lower and lower overstresses and the fulfillment of plastic consistency $(f=0)$ is progressively regained. As a consequence, the intermediate range $H_{\chi}-$ $\frac{\Phi}{d \Phi / d f} \mu_{\max }<H<H_{\chi}$ in Figure 3 tends to disappear, and the sign of the quadratic form in (45) is no longer affected by the rate vector $\mathbf{X}$. This mathematically translates that, in inviscid solids, the stress/strain rate has no influence on defining the current material state, nor on the triggering of constitutive instabilities.

\section{CONCLUDING REMARKS}

In this paper, a theoretical approach for the analysis of constitutive instabilities in elasto-viscoplastic solids has been proposed. At variance with previous works on the subject, general mixed loading conditions have been considered, accounting for the fact that in real laboratory tests and boundary value problems not all the stress or strain components are known/prescribed, but rather a combination of some of them. While the same problem was previously tackled by other authors for rate-insensitive elasto-plastic materials, a different approach has been followed here to overcome the lack of the tangent stiffness operator in Perzyna-type constitutive equations.

Under the assumption of isotropic/homothetic strain-hardening, it has been shown that instabilities are not possible while the hardening modulus is larger than the so-called controllability modulus $H_{\chi}$ - which is consistent with the rate-independent theory developed by [33]. While the scalar modulus $H_{\chi}$ contains information about the static state of the material (stresses and hardening variables) and the specific loading constraints, it has been also found that, below the $H_{\chi}$ limit, instabilities may occur depending on the current stress/strain rate. As a consequence, the latter actively contribute to define the global state of the material. It is worth remarking that, as the theory provides an 'instantaneous' condition, the actual development of macroscopic instability requires positive local accelerations to last over a sufficient amount of time.

The framework proposed in this paper extends the previous rate-independent theory and will enable to cope with relevant problems where time effects can play a major role.

\footnotetext{
** The yield function $f$ must be dimensionless.
} 


\section{ACKNOWLEDGEMENTS}

The authors gratefully thank Dr. Giuseppe Dattola for the valuable suggestions provided during the development of this research.

\section{APPENDIX A. ANALYTICAL DERIVATIONS}

The main analytical derivations skipped in Section 3.2 are hereafter reported.

Stress control $(\alpha=I, \beta=\varnothing)$

Under the stress control $\boldsymbol{\sigma}(t)=\boldsymbol{\Sigma}(t)$, the terms in Equation 15 can be specified as it follows:

$$
\begin{aligned}
\frac{d \Phi}{d t} & =\frac{d \Phi}{d f}\left(\frac{\partial f}{\partial \boldsymbol{\sigma}}^{T} \frac{d \boldsymbol{\sigma}}{d t}+\frac{\partial f^{T}}{\partial \boldsymbol{q}} \frac{\partial \boldsymbol{q}}{\partial \boldsymbol{\varepsilon}^{v p}} \frac{d \boldsymbol{\varepsilon}^{v p}}{d t}\right)= \\
& =\frac{d \Phi}{d f}{\frac{\partial f^{T}}{\partial \boldsymbol{\sigma}}}^{\boldsymbol{\Sigma}}+\Phi \frac{d \Phi}{d f} \frac{\partial f^{T}}{\partial \boldsymbol{q}} \frac{\partial \boldsymbol{q}}{\partial \boldsymbol{\varepsilon}^{v p}} \frac{\partial g}{\partial \boldsymbol{\sigma}}=-\Phi \frac{d \Phi}{d f} H+\frac{d \Phi}{d f}_{\frac{\partial f^{T}}{\partial \boldsymbol{\sigma}}} \dot{\boldsymbol{\Sigma}}
\end{aligned}
$$

and, exploiting the equality $\frac{d g}{d \sigma}=\frac{1}{\Phi} \frac{d \varepsilon^{v p}}{d t}$ (from the Perzyna's flow rule) along with Equation 16:

$$
\begin{aligned}
\frac{d \Phi}{d t} \frac{\partial g}{\partial \boldsymbol{\sigma}} & =-\Phi \frac{\partial \Phi}{\partial f} H \frac{\partial g}{\partial \boldsymbol{\sigma}}+\frac{d \Phi}{d f}\left(\frac{\partial f}{\partial \boldsymbol{\sigma}}^{T} \dot{\boldsymbol{\Sigma}}\right) \frac{1}{\Phi} \frac{d \boldsymbol{\varepsilon}^{v p}}{d t}= \\
& =-\frac{d \Phi}{d f} H \mathbf{I} \frac{d \boldsymbol{\varepsilon}^{v p}}{d t}+\frac{d \Phi}{d f} \frac{1}{\Phi}\left(\frac{\partial f}{\partial \boldsymbol{\sigma}}^{T} \dot{\boldsymbol{\Sigma}}\right) \mathbf{I} \frac{d \boldsymbol{\varepsilon}^{v p}}{d t}= \\
& =-\frac{d \Phi}{d f}\left(H+\frac{H_{\dot{\boldsymbol{\Sigma}}}}{\Phi}\right) \mathbf{I} \frac{d \boldsymbol{\varepsilon}}{d t}+\frac{d \Phi}{d f}\left(H+\frac{H_{\dot{\boldsymbol{\Sigma}}}}{\Phi}\right) \mathbf{C} \dot{\boldsymbol{\Sigma}} \\
\Phi \frac{d}{d t}\left(\frac{\partial g}{\partial \boldsymbol{\sigma}}\right)= & \Phi \frac{\partial^{2} g}{\partial \boldsymbol{\sigma} \otimes \partial \boldsymbol{\sigma}} \frac{d \boldsymbol{\sigma}}{d t}+\Phi \frac{\partial^{2} g}{\partial \boldsymbol{\sigma} \otimes \partial \boldsymbol{p}} \frac{\partial \boldsymbol{p}}{\partial \boldsymbol{\varepsilon}^{v p}} \frac{\partial \boldsymbol{\varepsilon}^{v p}}{\partial t}= \\
= & \Phi \frac{\partial^{2} g}{\partial \boldsymbol{\sigma} \otimes \partial \boldsymbol{\sigma}} \dot{\boldsymbol{\Sigma}}+\Phi \frac{\partial^{2} g}{\partial \boldsymbol{\sigma} \otimes \partial \boldsymbol{p}} \frac{\partial \boldsymbol{p}}{\partial \boldsymbol{\varepsilon}^{v p}} \frac{d \boldsymbol{\varepsilon}}{d t}-\Phi \frac{\partial^{2} g}{\partial \boldsymbol{\sigma} \otimes \partial \boldsymbol{p}} \frac{\partial \boldsymbol{p}}{\partial \boldsymbol{\varepsilon}^{v p}} \mathbf{C} \dot{\boldsymbol{\Sigma}}
\end{aligned}
$$

The aforementioned relationships are then substituted into Equation 17:

$$
\begin{gathered}
\frac{d^{2} \boldsymbol{\varepsilon}}{d t^{2}}=\mathbf{C} \ddot{\boldsymbol{\Sigma}}-\frac{d \Phi}{d f}\left(H+\frac{H_{\dot{\boldsymbol{\Sigma}}}}{\Phi}\right) \mathbf{I} \frac{d \boldsymbol{\varepsilon}}{d t}+\Phi \frac{\partial^{2} g}{\partial \boldsymbol{\sigma} \otimes \partial \boldsymbol{p}} \frac{\partial \boldsymbol{p}}{\partial \boldsymbol{\varepsilon}^{v p}} \frac{d \boldsymbol{\varepsilon}}{d t}+ \\
+\frac{d \Phi}{d f}\left(H+\frac{H_{\dot{\Sigma}}}{\Phi}\right) \mathbf{C} \dot{\boldsymbol{\Sigma}}+\Phi \frac{\partial^{2} g}{\partial \boldsymbol{\sigma} \otimes \partial \boldsymbol{\sigma}} \dot{\boldsymbol{\Sigma}}-\Phi \frac{\partial^{2} g}{\partial \boldsymbol{\sigma} \otimes \partial \boldsymbol{p}} \frac{\partial \boldsymbol{p}}{\partial \boldsymbol{\varepsilon}^{v p}} \mathbf{C} \dot{\boldsymbol{\Sigma}}
\end{gathered}
$$

and the final form (18) is readily obtained.

Strain control $(\alpha=\varnothing, \beta=I)$

Under the strain control $\varepsilon(t)=\boldsymbol{E}(t)$, the combination of Equations 15 and (23) gives rise to the following terms:

$$
\frac{d \Phi}{d t}=\frac{d \Phi}{d f}\left(\frac{\partial f}{\partial \boldsymbol{\sigma}}^{T} \frac{d \boldsymbol{\sigma}}{d t}+\frac{\partial f}{\partial \boldsymbol{q}}^{T} \frac{\partial \boldsymbol{q}}{\partial \boldsymbol{\varepsilon}^{v p}} \frac{d \boldsymbol{\varepsilon}^{v p}}{d t}\right)=\frac{d \Phi}{d f}\left(\frac{\partial f}{\partial \boldsymbol{\sigma}}^{T} \frac{d \boldsymbol{\sigma}}{d t}-\Phi H\right) \Longrightarrow
$$




$$
\begin{aligned}
\Longrightarrow-\mathbf{D} \frac{d \Phi}{d t} \frac{\partial g}{\partial \boldsymbol{\sigma}} & =-\frac{d \Phi}{d f}\left[\mathbf{D} \frac{\partial f^{T}}{\partial \boldsymbol{\sigma}} \frac{d \boldsymbol{\sigma}}{d t} \frac{\partial g}{\partial \boldsymbol{\sigma}}-H \mathbf{D}\left(\dot{\boldsymbol{E}}-\mathbf{C} \frac{d \boldsymbol{\sigma}}{d t}\right)\right]= \\
& =-\frac{d \Phi}{d f}\left[\mathbf{D} \frac{\partial f^{T}}{\partial \boldsymbol{\sigma}}\left(\mathbf{D} \dot{\boldsymbol{E}}-\mathbf{D} \Phi \frac{\partial g}{\partial \boldsymbol{\sigma}}\right) \frac{1}{\Phi}\left(\dot{\boldsymbol{E}}-\mathbf{C} \frac{d \boldsymbol{\sigma}}{d t}\right)-H \mathbf{D} \dot{\boldsymbol{E}}+H \mathbf{I} \frac{d \boldsymbol{\sigma}}{d t}\right]= \\
& =-\frac{d \Phi}{d f}\left[-\frac{H_{\dot{E}}}{\Phi} \mathbf{D} \dot{\boldsymbol{E}}+H_{c} \mathbf{D} \dot{\boldsymbol{E}}+\frac{H_{\dot{E}}}{\Phi} \mathbf{I} \frac{d \sigma}{d t}-H_{c} \mathbf{I} \frac{d \sigma}{d t}-H \mathbf{D} \dot{\boldsymbol{E}}+H \mathbf{I} \frac{d \boldsymbol{\sigma}}{d t}\right]= \\
& =-\frac{d \Phi}{d f}\left(\frac{H_{\dot{E}}}{\Phi}+H-H_{c}\right) \mathbf{I} \frac{d \sigma}{d t}+\frac{d \Phi}{d f}\left(\frac{H_{\dot{E}}}{\Phi}+H-H_{c}\right) \mathbf{D} \dot{\boldsymbol{E}}
\end{aligned}
$$

and

$$
\begin{gathered}
\Longrightarrow-\mathbf{D} \Phi \frac{d}{d t}\left(\frac{\partial g}{\partial \boldsymbol{\sigma}}\right)=-\Phi \mathbf{D}\left[\frac{\partial^{2} g}{\partial \boldsymbol{\sigma} \otimes \partial \boldsymbol{\sigma}} \frac{d \boldsymbol{\sigma}}{d t}+\frac{\partial^{2} g}{\partial \boldsymbol{\sigma} \otimes \partial \boldsymbol{p}} \frac{\partial \boldsymbol{p}}{\partial \boldsymbol{\varepsilon}^{v p}}\left(\dot{\boldsymbol{E}}-\mathbf{C} \frac{d \boldsymbol{\sigma}}{d t}\right)\right]= \\
-\Phi \mathbf{D}\left(\frac{\partial^{2} g}{\partial \boldsymbol{\sigma} \otimes \partial \boldsymbol{\sigma}}-\frac{\partial^{2} g}{\boldsymbol{\sigma} \otimes \partial \boldsymbol{p}} \frac{\partial \boldsymbol{p}}{\partial \boldsymbol{\varepsilon}^{v p}} \mathbf{C}\right) \frac{d \boldsymbol{\sigma}}{d t}-\Phi \mathbf{D} \frac{\partial^{2} g}{\partial \boldsymbol{\sigma} \otimes \partial \boldsymbol{p}} \frac{\partial \boldsymbol{p}}{\partial \boldsymbol{\varepsilon}^{v p}} \dot{\boldsymbol{E}}
\end{gathered}
$$

which can easily be recast in the compact form (24).

\section{Mixed stress-strain control}

Because mixed control conditions involve both stress and strain controls, the ODE system (35) can be derived by wisely combining the analytical techniques employed earlier for both the pure stress and pure strain control cases. Reporting here all the details does not seem to be particularly instructive; the partitioned structure of the generalized Perzyna's relationship (31) is just to be carefully handled. The analytical derivations can be significantly simplified if the assumption (36) (isotropic-homothetic strain-hardening) is retained from the beginning.

\section{APPENDIX B. SPECTRAL PROPERTIES OF THE MATRIX A}

\section{- All the eigenvalues of $\mathbf{A}$ are real and semisimple}

According to standard matrix algebra, the eigenvalues of an $n \times n$ matrix are defined as semisimple if their algebraic $\left(m_{\text {alg }}\right)$ and geometrical $\left(m_{\text {geo }}\right)$ multiplicities coincide $\left(m_{g e o}\right.$ is the dimension of the associated eigenspace). As was stated in Section 4.2, the peculiar structure of A (Figure 2) implies:

$$
\Lambda(\mathbf{A})=\Lambda\left(\mathbf{A}_{\alpha \alpha}\right) \cup \Lambda\left(\mathbf{A}_{\beta \beta}\right)
$$

As for $\Lambda\left(\mathbf{A}_{\alpha \alpha}\right)$, the eigenvalue $-\frac{d \Phi}{d f}\left(H-H_{\chi}\right)$ in (48) is such that $m_{\text {alg }}=m_{g e o}=|\alpha|$ because $\mathbf{A}_{\alpha \alpha}$ is proportional to $\mathbf{I}_{\alpha \alpha}$.

Conversely, the properties of $\Lambda\left(\mathbf{A}_{\beta \beta}\right)$ depend on the adopted $g$ function, but there is no general reason to infer the existence of eigenvalues with $m_{\text {alg }}>1$. Unless for very particular cases, $\mathbf{A}$ has $|\beta|+1$ semisimple real eigenvalues.

\section{- Eigenvalues of $\mathbf{A}_{\beta \beta}$}

It is possible to determine the sign of the eigenvalues $\lambda_{\beta \beta}^{i}$ on the basis of expression (49).

While the elastic compliance matrix $\mathbf{C}$ (and its inverse too) is positive definite to guarantee positive elastic strain energy, the Hessian matrix $\frac{\partial^{2} g}{\partial \sigma \otimes \partial \sigma}$ generated by the plastic potential $g$ is also positive definite on condition that $g$ is strictly convex in the stress space - which is the case of most constitutive relationships. 
Then, it can be proven that $\mathbf{C}_{\beta \beta}^{-1}$ and $\frac{\partial^{2} g}{\partial \boldsymbol{\sigma}_{\beta} \otimes \partial \sigma_{\beta}}$ are sub-matrices from two matrices that are similar to $\mathbf{C}^{-1}$ and $\frac{\partial^{2} g}{\partial \boldsymbol{\sigma} \otimes \partial \boldsymbol{\sigma}}$, respectively (through the linear transformation needed to reorder the controlled/uncontrolled variables). Therefore, because (i) similarity transformations do not alter the spectrum and (ii) all the principal minors of symmetric positive definite matrices are positive (Sylvester's criterion), it turns out that $\mathbf{C}_{\beta \beta}^{-1}$ and $\frac{\partial^{2} g}{\partial \sigma_{\beta} \otimes \partial \sigma_{\beta}}$ are positive definite as well.

Provided that the product of symmetric positive definite matrices produces a positive definite matrix, it can be stated that the matrix $\mathbf{M}=\mathbf{C}_{\beta \beta}^{-1} \frac{\partial^{2} g}{\partial \sigma_{\beta} \otimes \partial \sigma_{\beta}}$ is positive definite. The latter observation and the positiveness of $\Phi$ (in the viscoplastic regime) prove that all the eigenvalues of $\Phi \mathbf{M}$ are strictly positive, so that all the eigenvalues in $\Lambda\left(\mathbf{A}_{\beta \beta}\right)$ are lower than $-\frac{d \Phi}{d f}\left(H-H_{\chi}\right)$.

\section{REFERENCES}

1. Bažant ZP, Cedolin L. Stability of Structures: Elastic, Inelastic, Fracture and Damage Theories (3rd edn). World Scientific: Singapore, 2010.

2. Daouadji A, Darve F, Al Gali H, Hicher PY, Laouafa F, Lignon S, Nicot F, Nova R, Pinheiro M, Prunier F, Sibille L, Wan R. Diffuse failure in geomaterials: experiments, theory and modelling. International Journal for Numerical and Analytical Methods in Geomechanics 2011; 35(16):1731-1773.

3. Darve F, Vardoulakis I. Degradations and Instabilities in Geomaterials, Vol. 461. Springer: Berlin, 2005.

4. Sulem J, Vardoulakis I. Bifurcation Analysis in Geomechanics. Chapman \& Hall: London, 1995.

5. Chambon R. Some theoretical results about second-order work, uniqueness, existence and controllability independent of the constitutive equation. In Mathematics and Mechanics of Granular Materials. Springer: Dordrecht, The Netherlands, 2005; 53-61.

6. Darve F, Servant G, Laouafa F, Khoa H. Failure in geomaterials: continuous and discrete analyses. Computer Methods in Applied Mechanics and Engineering 2004; 193(27):3057-3085.

7. Koiter WT. General Theorems for Elastic-plastic Solids. North-Holland: Amsterdam, 1960.

8. Lemaitre J, Chaboche J. Mechanics of Solid Materials. Cambridge University Press: Cambridge, 1990.

9. Lubliner J. Plasticity Theory. Mac Millan: New York, 1990.

10. Vermeer PA, De Borst R. Non-associated plasticity for soils, concrete and rock, 1984.

11. Duvaut G, Lions JL. Les Inéquations en Mécanique et en Physique, Vol. 18. Dunod: Paris, 1972.

12. Heeres OM, Suiker AS, de Borst R. A comparison between the perzyna viscoplastic model and the consistency viscoplastic model. European Journal of Mechanics-A/Solids 2002; 21(1):1-12.

13. Perzyna P. The constitutive equations for rate sensitive plastic materials. Quarterly of Applied Mathematics 1963; 20:321-332.

14. Perzyna P. Fundamental problems in viscoplasticity. Advances in Applied Mechanics 1966; 9:243-377.

15. Wang WM, Sluys LJ, de Borst R. Viscoplasticity for instabilities due to strain softening and strain-rate softening. International Journal for Numerical Methods in Engineering 1997; 40(20):3839-3864.

16. Adachi T, Oka F. Constitutive equations for normally consolidated clay based on elasto-viscoplasticity. Soils and Foundations 1982; 22(4):57-70.

17. Borja R, Kavazanjian E. A constitutive model for the stress-strain-time behaviour of 'wet' clays. Géotechnique 1985; 3:283-298.

18. Desai CS, Zhang D. Viscoplastic model for geologic materials with generalized flow rule. International Journal for Numerical and Analytical methods in Geomechanics 1987; 11(6):603-620.

19. di Prisco C, Imposimato S. Time dependent mechanical behaviour of loose sands. Mechanics of Cohesive-Frictional Materials 1996; 1(1):45-73.

20. Georgin J, Reynouard J. Modeling of structures subjected to impact: concrete behaviour under high strain rate. Cement \& Concrete Composites 2003; 25(1):131-143.

21. di Prisco C, Imposimato S. Experimental analysis and theoretical interpretation of triaxial load controlled loose sand specimen collapses. Mechanics of Cohesive-Frictional Materials 1997; 2(2):93-120.

22. Lade P, Yamamuro J, Bopp P. Influence of time effects on instability of granular materials. Computers and Geotechnics 1997; 20(3/4):179-193.

23. Oka F, Adachi T, Yashima A. Instability of an elasto-viscoplastic constitutive model for clay and strain localization. Mechanics of Materials 1994; 18:119-129.

24. Loret B, Prevost J. Dynamic strain localization in elasto-(visco-)plastic solids. Computer Methods in Applied Mechanics and Engineering 1990; 83(3):247-273.

25. Loret B, Prevost J. On the existence of solutions in layered elasto-(visco-)plastic solids with negative hardening. European Journal of Mechanics. A/Solids 1991; 10:575-586.

26. Needleman A. Material rate dependence and mesh sensitivity in localization problems. Computer Methods in Applied Mechanics and Engineering Archive 1988; 67(1):69-85.

27. Anand L, Kim K, Shawki T. Onset of shear localization in viscoplastic solids. Journal of the Mechanics and Physics of Solids 1987; 35(4):407-429. 
28. Shawki T, Clifton R. Shear band formation in thermal viscoplastic materials. Mechanics of Materials 1989; 8(1): $13-43$.

29. di Prisco C, Imposimato S, Vardoulakis I. Mechanical modelling of drained creep triaxial tests on loose sand. Géotechnique 2000; 50(1):73-82.

30. Oka F, Adachi T, Yashima A. A strain localization analysis using a viscoplastic softening model for clay. International Journal of Plasticity 1995; 11:523-545.

31. Imposimato $S$, Nova R. An investigation on the uniqueness of the incremental response of elastoplastic models for virgin sand. Mechanics of Cohesive-frictional Materials 1998; 3(1):65-87.

32. Nova R. Controllability of the incremental response of soil specimens subjected to arbitrary loading programmes. Journal of the Mechanical Behavior of Materials 1994; 5(2):193-202.

33. Buscarnera G, Dattola G, di Prisco C. Controllability, uniqueness and existence of the incremental response: a mathematical criterion for elastoplastic constitutive laws. International Journal of Solids and Structures 2011; 48(13): $1867-1878$.

34. Bigoni D. Nonlinear Solid Mechanics: Bifurcation Theory and Material Instability. Cambridge University Press: New York, 2012.

35. Bonelli S, Dascalu C, Nicot F. Advances in Bifurcation and Degradation in Geomaterials: Proceedings of the 9th International Workshop on Bifurcation and Degradation in Geomaterials, Vol. 11. Springer Science \& Business Media: New York, 2011.

36. Chambon R, Caillerie D, Viggiani G. Loss of uniqueness and bifurcation vs instability: some remarks. Revue Française de Génie Civil 2004; 8(5-6):517-535.

37. Petryk H. Theory of Material Instability in Incrementally Nonlinear Plasticity. Springer: Wien, 2000.

38. Darve F. Une formulation incrémentale des lois rhéologiques, application aux sols. Ph.D. Thesis, Institut de Mécanique de Grenoble, 1978.

39. Darve F, Labanieh S. Incremental constitutive law for sands and clays: simulations of monotonic and cyclic tests. International Journal for Numerical and Analytical Methods in Geomechanics 1982; 6(2):243-275.

40. Hill R. A general theory of uniqueness and stability in elastic-plastic solids. Journal of the Mechanics and Physics of Solids 1958; 6(3):236-249.

41. Maier G, Hueckel T. Non-associated and coupled flow rules of elastoplasticity for rock-like materials. International Journal of Rock Mechanics and Mining Sciences 1979; 16(2):77-92.

42. Maier G. Sui legami associati tra sforzi e deformazioni incrementali in elastoplasticita. Istituto Lombardo, Rendiconti, Classe di Scienze 1966.

43. Ju J. Consistent tangent moduli for a class of viscoplasticity. Journal of Engineering Mechanics 1990; 116(8): 1764-1779.

44. Wood DM. Geotechnical Modelling, Vol. 1. Taylor \& Francis: New York, 2003.

45. Lyapunov AM. The general problem of motion stability. Kharkovskoye Matematicheskoe Obshchestvo $1892 ; 11$.

46. Seydel R. From Equilibrium to Chaos. Elsevier: New York, 1988.

47. Freitas T, Potts D, Zdravkovic L. Implications of the definition of the $\Phi$ function in elastic-viscoplastic models. Géotechnique 2012; 62(7):643-648. 